\title{
BDA slams NHS charge hike in middle of pandemic
}

The British Dental Association (BDA)

has slammed the latest inflation-busting

$5 \%$ increase in NHS dental charges

in England announced in November,

accusing government of erecting further barriers to care during the COVID

pandemic, when services remain so

limited and focused on dealing with an

unprecedented backlog.

The hike means:

- A routine check-up will increase by

$£ 1.10$ from $£ 22.70$ to $£ 23.80$

- Treatments such as root canals or removing teeth will rise by $£ 3.10$ from $£ 62.10$ to $£ 65.20$

- More complex procedures like crowns, dentures and bridges rise from by $£ 13.50$ from $£ 269.30$ to $£ 282.80$.

The fee increase, originally slated for 1 April, was postponed by the first wave of the pandemic, and will now take effect as of 14 December 2020.

The BDA estimates over 19 million appointments have been delayed since lockdown. The BDA has long warned that costs are a major barrier to patients, even among those who are entitled to free care. Analysis of data from the government's GP Survey indicates over 700,000 people had avoided seeking NHS treatment for reason of cost. The Adult Oral Health Survey has found that for $26 \%$ of the public, cost had influenced the type of dental treatment they had opted to have and 19\% had delayed dental treatment due to cost.

Dentist leaders have accused ministers of using inflation-busting increases to try and plug the hole in the service's shrinking budget. Since lockdown the Treasury has lost nearly $£ 400$ million from the charges that are increasingly relied upon to fund services in England, with around $£ 50$ million in revenues now being lost per month. The BDA has already called on government to provide capital funding for ventilation equipment that could enable practices to massively expand access, by reducing the gaps dentists are mandated to keep between treatments, which remain the number one barrier to capacity. The BDA estimate any investment would pay for itself by helping restore patient numbers closer to preCOVID levels.

Dentistry is the only part of the NHS operating on a lower budget than it received in 2010, with no effort made to keep pace with the cost of living or population growth. Prior to the pandemic real terms net government contributions have fallen by over $£ 650$ million since 2010, while charge levels have increased by over $40 \%$ to plug the gap. In Wales fees have increased by around just $13 \%$ in the same period. 